\title{
Prosthodontic rehabilitation of a xerostomic edentulous patient - A case report
}

\author{
Pulkit Chandra' $^{1, *}$, Siddharth Mehta ${ }^{2}$, Arpit J. Halani ${ }^{3}$, Sadhana Gupta ${ }^{4}$ \\ ${ }^{1,2}$ Senior Lecturer, Dept. of Prosthodontics, ${ }^{3}$ Consultant Prosthodontist, ${ }^{4}$ Deputy Director, ${ }^{1}$ Jaipur Dental College, Jaipur, \\ Rajasthan, ${ }^{2}$ Bapuji Dental College and Hospital, Davangere, Karnataka, ${ }^{4}$ Zanana Hospital, Jaipur, Rajasthan, India
}

*Corresponding Author:

Email: chandra.pulkit@gmail.com

\begin{abstract}
Xerostomia is a clinical condition caused by a reduced secretion of saliva in the oral cavity. Xerostomia in itself is not a disease but rather a manifestation of injury/destruction of the salivary glands by some disease or radiation therapy. As seen in today's world most of the xerostomic cases seen are patients who have received radiotherapy in the head and neck area for treating one or the other form of cancer, which causes damage to the glandular function and structure of the major and minor salivary glands. Multiple root caries, fungal infections, impairment of speech and difficulties in chewing and swallowing food are some of the many complications that are caused by the reduced salivary production in the oral cavity. The difficulty level raises many folds if the patient is edentulous and is/will be a denture wearer due to soreness and frequent ulceration of the mucosa. As far as the solutions to this problem are concerned, many intra-oral devices /appliances such as the salivary reservoir in denture patients, have been tried which allow the slow release of artificial salivary substitutes into the oral cavity to provide some kind of relief to the patient. However most of the techniques present in the literature for the fabrication of a salivary reservoir either make the denture extremely bulky or quite expensive for the general public to afford. Therefore in this case we have described a technique that is simple, cost effective and novel, illustrating the incorporation of a salivary reservoir in a maxillary denture with salivary substitute along with the management of a resorbed mandibular edentulous ridge.
\end{abstract}

Keywords: Xerostomia, Reservoir denture, Salivary substitutes, Complete denture, Radiotherapy, Neutral zone, Resorbed ridge.

\section{Introduction}

Dryness of the mouth from the lack of normal secretions is called Xerostomia, as elaborated in the Glossary of Prosthodontic terms. ${ }^{1}$

A whole variety of factors can lead to xerostomia such as Drugs, anxiety, depression, radiotherapy and some auto-immune conditions. ${ }^{2}$ As far as drugs are concerned everyday a new drug is highlighted which may be a causative factor for dry mouth but namely drugs such as anticholinergics, antihistamines, antihypertensive, antidepressants, many sedatives and drugs used in parkinsonism are usually the culprits affecting the salivary production. Another important aspect is the salivary flow which may be affected by certain mouthwashes, alcohol and smoking. ${ }^{3}$

Sjogren's syndrome and rheumatoid arthritis are some of the auto-immune conditions that may be the causative factors for xerostomia in some cases. ${ }^{4}$

Very often severe hypo-salivation is seen in patients receiving head and neck radiotherapy due to irreversible glandular tissue damage.

This specially in the context of denture patients is important because xerostomia may lead to mucositis, soreness, painful ulceration, difficulties in chewing and swallowing food and retention difficulties. ${ }^{5}$

The role of saliva in the normal functioning and maintenance of the oral hard and soft tissues is critical. The functions of the saliva are varied which include:

a. Lubrication - for proper speech and swallowing of food bolus and mastication

b. Maintenance of mucous membrane integrity

c. pH buffering d. debridement of food particles from the teeth

e. inhibit/minimize bacterial adhesions

f. Defence with lysozymes, lactoferrins, antifungals and salivary peroxidases etc.

g. Digestion - with salivary enzymes.

Pondering upon the treatment options medications such as Sialogogues (Pilocarpine) have been used to stimulate the salivary flow rate whenever, there is residual salivary gland function left. In the total absence of salivary glandular function the symptomatic management assumes a major role wherein salivary substitutes such as carboxymethylcellulose preparations, K-Y jelly and Oral Balance gel are commonly used.

Saliva substitutes to an extent improve the patient's quality of life. Their actions however are brought about by the use of Intra-oral devices/ reservoirs that have been developed to allow the slow release of saliva substitute into the mouth. Many such successful attempts have been documented in the literature ${ }^{5-12}$, which elaborate that a saliva reservoir is a chamber incorporated into a removable prosthesis that holds and provides a flow of salivary substitute for a certain period of time. Saliva reservoirs range in volume from $2.3 \mathrm{ml}$ to $5.3 \mathrm{ml}$ and provide a flow of artificial saliva for 2 to 5 hours. ${ }^{6}$

Here, we discuss the prosthetic rehabilitation of a xerostomic patient with resorbed mandibular ridge. 


\section{Case Report}

A 45 year old female patient reported to the department of prosthodontics with a chief complain of difficulty in eating and chewing due to missing teeth and difficulty in swallowing due to reduced amount of saliva. She did not have any history of any previous dentures. Medical history revealed that she had received radiotherapy in the head and neck region for a neoplastic lesion.

Extra-oral examination revealed ovoid facial form with a convex facial profile with a reduced lip support. TMJ was found to be normal without any clicking or deviation on mouth opening. General health and physique of the patient was weak and thin.

Intra-oral examination revealed resorbed residual alveoloar ridges, severe in case of mandibular ridge. Also the palatal form was found to be v-shaped.

Oral mucosa was found to be dry and fragile. The 'mouth mirror sticking to mucosa' test was found to be positive, due to the lack of salivation.

Keeping in mind the condition of the ridges and the mucosa it was decided to fabricate a salivary reservoir within the maxillary complete denture and a mandibular denture using neutrocentric concept of occlusion to maximize the stability of the dentures.

Irreversible hydrocolloid was used to make the diagnostic impressions for the patient as the mucosa was too fragile and dry for using impression compound.

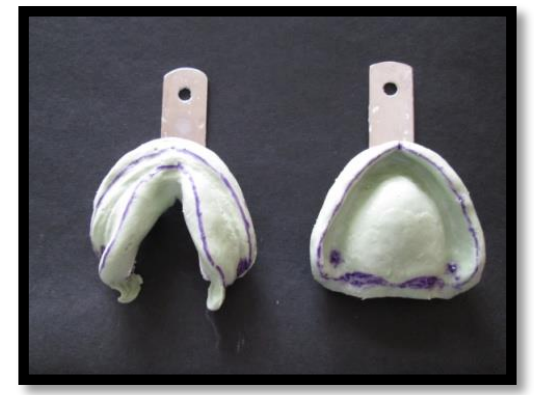

Fig. 1: Diagnostic Impressions

Special trays were fabricated and single step border moulding was done using Polyvinyl siloxane putty material following which final impression were made using light body impression material.

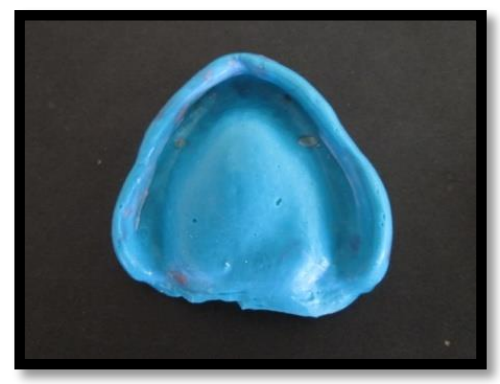

Fig. 2: Final Impression (Maxillary arch)

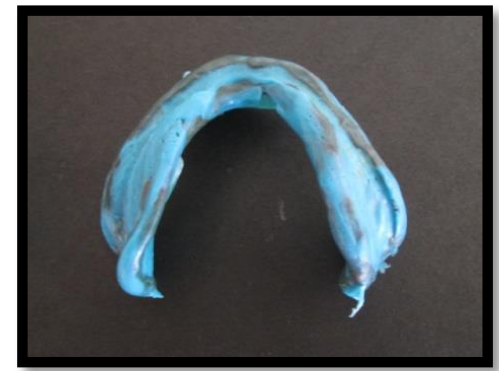

Fig 3: Final impression (Mandibular arch)

Temporary record bases were fabricated and the maxillary rim was customized and mounted on the articulator using face-bow transfer.

Following this compound occlusal rims were fabricated using a mix of impression compound and green stick compound and adjusted in the patient's mouth to record the neutral zone. Patient was instructed to perform certain movements like swallowing, moving the tongue from side to side and sucking, pursing the cheeks and speaking letters such as ' $\mathrm{E}$ ' and ' $\mathrm{O}$ ' repeatedly. The rims were removed, softened and the actions were carried out again till there was no change in the shape of the compound rims, following which a wash impression of the rims was recorded with light body impression material. The rationale behind this was to locate the zone where the forces of the lips, cheeks and tongue are balanced with each other i.e. the Neutral zone.

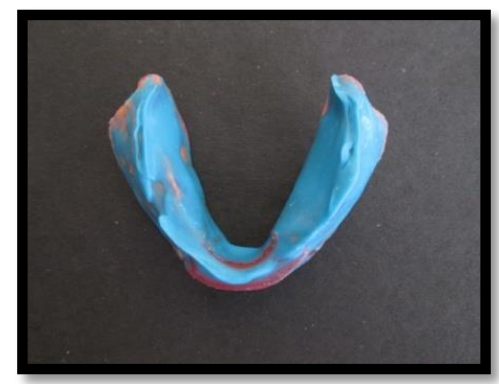

Fig. 4: Occlusal rim with recorded Neutral zone

The rims were removed and the putty indices were fabricated based on the shape of the muscle trimmed rims. The compound rims were removed and replaced with regular modeling wax and adjusted according to the putty indices. The pending jaw Relations were recorded along with Facebow transfer. The teeth arrangement was completed using standard protocol. Care was taken to ensure that the teeth were arranged in the neutral zone by checking with the putty indices. Try-in was performed and patient acceptance was obtained. 


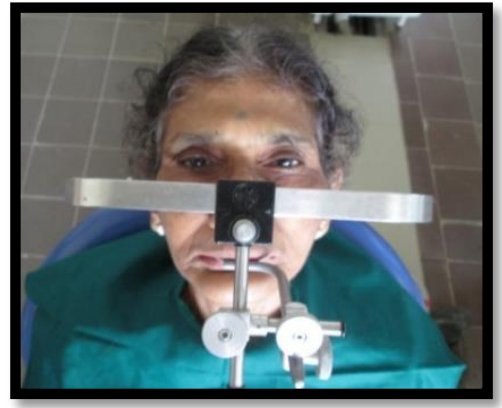

Fig 5: Facebow Transfer

\section{Fabrication of the Salivary Reservoir}

An impression for shaping the cameo surface of the palatal part of the maxillary denture was made with tissue conditioner.

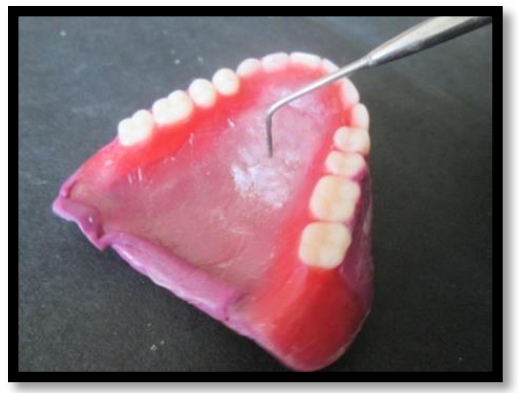

Fig 6: Impression using tissue conditioner

A cast was poured using this impression for the fabrication of the acrylic framework of the salivary reservoir using the traditional wax up and acrylization process (Fig 7).

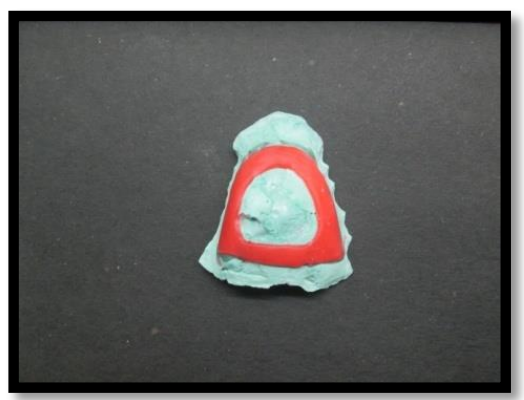

Fig 7: Wax pattern for Acrylic framework

A Thermplastic sheet was adapted on the master cast which in-turn was used to make the floor of the salivary reservoir. After the dentures were acrylized, the acrylic framework and the adapted thermoplastic sheet were joined to the maxillary denture using selfcure acrylic resin.

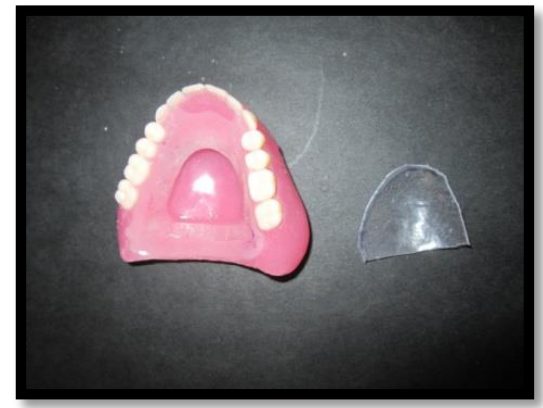

Fig 8: Maxillary Denture and the Acrylic framework with moulded thermoplastic sheet

The dentures were finished and polished and artificial saliva was filled in the reservoir space using a syringe. The outlet holes were prepared both on the tissue and the cameo surface of the denture. The dentures were inserted in the oral cavity and the patient was immediately able to appreciate the difference in the mouth due to the presence of teeth and the soothing effect of artificial saliva present. The patient was instructed about the denture hygiene and refilling and maintenance of the reservoir. She was kept on regular follow up visits and did not report any problems with the dentures and was satisfied.

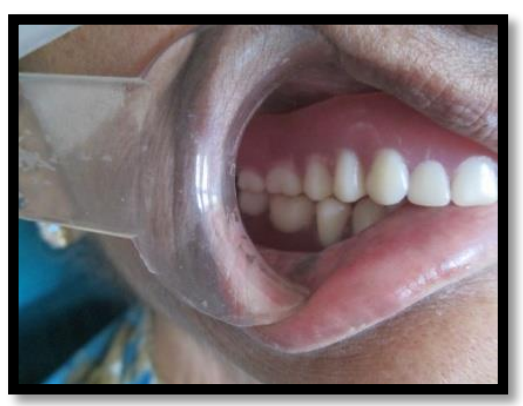

Fig 9: Occlusion: Right lateral view

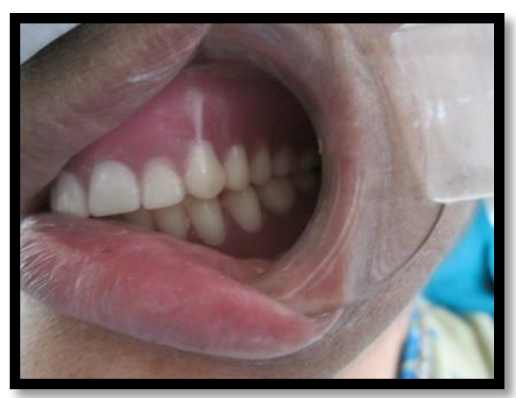

Fig 10: Occlusion: Left lateral view 


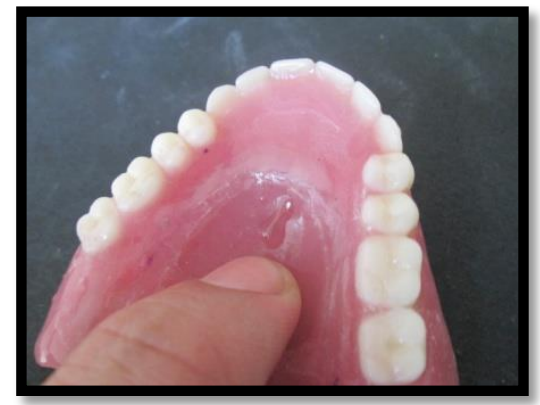

Fig 11: Denture with Artificial Saliva

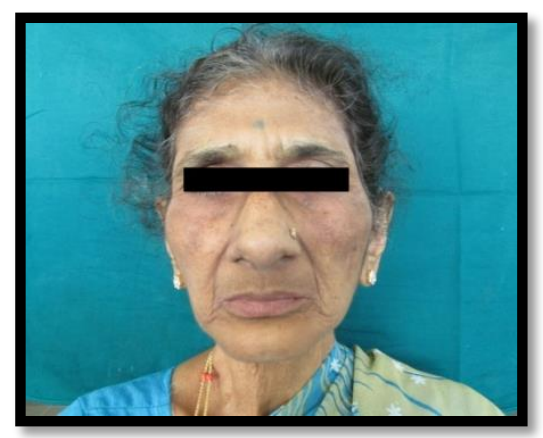

Fig. 13: Before treatment

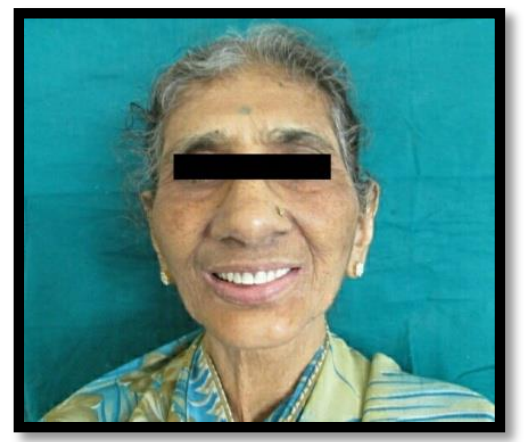

Fig. 14: After treatment

\section{Discussion}

After standard head and neck irradiation for nasopharyngeal carcinomas, salivary hypofunction is an almost ubiquitous lifelong complication for the patients and clinicians. ${ }^{4}$ Symptoms in the patient with salivary gland hypofunction are related to decreased fluid in the oral cavity. Patients complain of dryness of all the oral mucosal surfaces, including the throat and also of difficulty in chewing, swallowing and speaking. The mucosa may also become sensitive to spicy or coarse foods. $^{2}$

Saliva substitutes containing thickening agents for longer relief and increased moistening and lubrication of the oral surfaces have been developed. These are available as solutions, sprays or gels and have multiple contents such as carboxymethylcellulose, electrolytes and flavoring agents e.g. wet mouth (ICPA Health Products Ltd), aqwet (Cipla Ltd), saleva (DentAids Ltd.). ${ }^{7}$ However, the main problem is to deliver this substitute constantly into patient's mouth without affecting their normal routine. Where all treatment modalities have proven unsuccessful, the incorporation of artificial salivary reservoir in dentures, has been proposed in such cases. ${ }^{5-12}$

This paper presents the problems and their management in the rehabilitation of a radiation induced xerostomic patient using a novel technique.

\section{Problems}

1. Fragile and sore oral mucosa: Causes difficulty in impression making which was managed by using irreversible hydrocolloid impression material for the primary impressions and elastomers for the final impressions rather than opting for impression compound and Zinc oxide eugenol impression pastes.

2. Frequent ulceration of the oral mucosa: Leads to painful wearing of the denture, sometimes to an extent that the patient refuses to wear the dentures. This was managed with artificial salivary substitutes which lubricated and reduced the amount of friction between the dentures and oral mucosa.

3. Reduced retention of the dentures: The slow release of the salivary substitute along with proper occlusion with non-deflective contacts in centric and eccentric positions within the neutral zone minimize such difficulties.

4. Psychological issues: These may be related to the trauma that the patient has gone through during the radiotherapy or denture related both these situations to a great extent can be managed with good patient counselling and regular follow up visits.

Specifically, talking about the benefits of incorporating a palatal salivary reservoir in the dentures include

a. A quick and simple technique

b. Minimal complications and maintenance issues due to the absence of any detachable parts leading to longevity with minimal hassles.

c. Easy maintenance and cleaning due to the palatal inlet and outlet holes present in the denture and the use of a natural pumping mechanism.

d. Easy refill and visualization of the level of the salivary substitute in the reservoir through the translucent membrane.

e. Cost effective: The thermoplastic membrane that has been used can be easily replaced by the clinician chair side requiring a minimum amount of time and materials.

f. Relining can be done easily with reproduction of the palatal reservoir.

Mechanism of working for the salivary reservoir: This reservoir utilizes swallowing as a control mechanism for the flow of salivary substitute. As the patient swallows, the tongue contacts the anterior part of the 
palate and the membrane which creates positive pressure inside the reservoir thus pushing the salivary substitute out of the outlets both on the tissue surface and the cameo surfaces of the denture to ensure good wetting of the oral mucosa. When the pressure is relieved, air is sucked in creating a negative pressure and again the next cycle starts.

The overall thickness of the palate was increased which led to an initial difficulty in speech such as difficulty in pronunciation of $\mathrm{ch} /, / \mathrm{s} /$, and /sh/ syllables, however within a period of 15 days the patient got used to the thickness and this difficulty subsided.

Apart from providing a reservoir denture, the patient was requested to make a conscious effort to consume at least 8-10 glasses of water, juice or milk daily. Additional recommendations were to include beverages that may produce more saliva such as water with a slice of lemon or lemonade. ${ }^{3}$

\section{Conclusion}

In management of patients suffering with post radiotherapy and psychological complications it is imperative that we keep a comforting and supportive attitude. It should be kept in mind that the choice of treatment must be such which involves minimum time and patient visits and provides a durable well functioning prosthesis. Incorporation of multiple movable or battery operated devices in such cases may sometimes lead to repeated breakage or replacements and a distressed patient.

Funding: No funding sources.

Conflict of interest: None declared.

\section{References}

1. The glossary of prosthodontics terms. J Prosthet Dent 2005;94:10-92.
2. Martin S. Greenberg, Michael Glick. Burket's Oral Medicine Diagnosis \& Treatment.10th ed. Elsvier; 2003;236-37.

3. Zarb GA, Bolender CL, Eckert SE, Fenton AH, Jacob RF, Mericske-Stern R. Prosthodontic treatment for edentulous patients. 12th ed. St. Louis: Mosby 2004. p. 27,57-8.

4. Anne S. McMillan, C.S. Peter Tsang, May C.M. Wong, Andrew Y.L. Kam. Efficacy of a novel lubricating system in the management of radiotherapy-related xerostomia. Oral Oncol (2006);1- 7.

5. Mendosa AR, Tomlinson MJ. The split denture: a new technique for artificial saliva reservoirs in mandibular dentures. Aust Dent J 2003;48:190-94.

6. Upadhyay Snehal Rashmikant, Kumar Lakshya, and RaoJitendra. Fabrication of a functional palatal saliva reservoir by using a resilient liner during processing of a complete denture. J Prosthet Dent 2012;108:332-35.

7. Pattanaik Bikash, Pattanaik Seema. Prosthetic Rehabilitation of A Xerostomia Patient with A Mandibular Split Salivary Reservoir Denture. Ann Essence Dent 2010.

8. Vergo TJ Jr, Kadish SP. Dentures as artificial saliva reservoirs in the irradiated edentulous cancer patient with xerostomia: a pilot study. Oral Surg Oral Med Oral Pathol 1981;51:229-33.

9. Sinclair GF, Frost PM, Walter JD.New design for an artificial saliva reservoir for the mandibular complete denture. J Prosthet Dent 1996;75:276-80.

10. Toljanic JA, Zucuskie TG.Use of a palatal reservoir in denture patients with xerostomia. J Prosthet Dent 1984;52:540-44

11. R. B. Hallikerimath, Manish Jain. Managing the Edentulous Dry Mouth: The Two Part Mandibular Denture. J Indian Prosthodont Soc 2012;12(1):51-4.

12. Nupur Dabas, Sumit Singh Phukela, and Harish Yadav. The Split Denture: Managing Xerostomia in Denture Patients: A Case Report. J Indian Prosthodont Soc 2011;11(1):67-70.

How to cite the article: Chandra P., Mehta S., Halani A., Gupta S. Prosthodontic rehabilitation of a xerostomic edentulous patient - A case report. Ann Prosthodont Restor Dent 2018;4(3):100-104. 